\title{
TECNICAS DE CONSERVACION DE LOS RECURSOS PESQUEROS EN LA AMAZONIA PERUANA
}

\author{
CORTEZ SOLIS JUAN PEDRO ( $\left.{ }^{*}\right)$
}

\section{INTRODUCCION}

La necesidad de conservar el excedente de pescado no utilizado en la alimentación diaria, por el problema que significaba la descomposición del pescado fresco y el tener que pescar mas seguido para compensarlo, dio lugar al empleo de los primeros métodos de conservación, que se presume hayan sido el secado, salado y ahumado; posteriormente, el pescador o recolector al ampliar su área de pesca, tuvo que experimentar métodos mejores, hasta llegar finalmente a la utilización del frio como elemento de preservación (cámaras de refrigeración, refrigeración con hielo y congelación).

En la amazonía peruana, los pescadores, principalmente los de pesca artesanal, para aprovechar la abundancia de capturas que se da en la época de vaciante, utilizan desde tiempos inmemoriales diversos métodos de conservación que les permite mantener su frescura por ciertos períodos de tiempo, hasta su consumo 0 comercialización.

La pesquería comercial, que es la principal fuente de abastecimiento de pescado fresco a los grandes mercados de la amazonia (Iquitos, Pucallpa y Y urimaguas), utiliza en cambio del sistema de preservación de refrigeración con hielo en trozos, que les permite desplazarse a areas más distantes de pesca.

El presente trabajo ha sido elaborado en base a los datos obtenidos en la primera fase del proyecto: Estudio de las técnicas de extracción y conservación de los recursos pesquero, que fue ejecutado en 1985 - 1986, cuyo objetivo principal consistió en lograr un diagnóstico situacional de los métodos actuales de extracción de los recursos pesqueros utilizados en la amazonia peruana.

Ingo Pesquero - Investinador: DIRECCION DE INVFSTIGACION

EN RECURSOS HIDROBIOLOGICOS

INSTITUTO DE INVESTIGACIONES DE LA AMAZONIA, PERUANA. 


\section{MATERIAL Y METODOS}

EI presente estudio se realizó en las principales áreas pesqueras de los ríos U cayali, M arañón, Huallaga, A mazonas y $\mathrm{N}$ apo.

Se consideró de importancia las técnicas de conservación que realiza el pescador artesanal (salado, salpreso, seco-salado) y la que se realiza en la pesca comercial.

Se participó en los procesos de conservación utilizados en los diferentes ríos.

Se utilizó cuchillas, bandejas plásticas, cajas de tecnoport y termos rectangulares.

EI desplazamiento se hizo en deslizadores y a embarcaciones pesqueras.

\section{RESULTADOS}

\section{A. Preservación del pescado fresco}

En la amazonía peruana se emplean dos (2) formas de preservación del pescado fresco: una, que trata de mantener el pescado con todas sus características iniciales de frescura, lo que se da específicamente en la pesca artesanal y otra, mediante la conservación por refrigeración, que es característica de la pesquería comercial.

\section{Pescado fresco a bordo}

Se da específicamente en la pesca de tipo artesanal, cuyas zonas de pesca están relativamente cerca al lugar de desembarque, aproximadamente de 2 a 4 horas en canoa y botes, donde para mantener el pescado con sus características de frescura durante el tiempo que dura las operaciones de captura y el transporte se utilizan artificios simples pero muy prácticos y efectivos a nivel de pesca menor.

En los ríos Ucayali y Marañón se observó el uso de "rapichero" para mantener y transportar peces vivos. En los ríos Napo y A mazonas se utilizan bolsas de plástico, así como redes de nylon (pedazos de boliches) colgados de las ramás de los árboles y semi-introducidos en el agua, con el fin de mantener a los peces vivos durante el tiempo que dura las faenas de pescado. Se emplea especialmente con peces medianos y menores. 
Para el transporte generalmente se estiba al granel el pescado en el mismo bote o canoa y se cubre con hojas de bijao, yarina, shapaja y plátano, que actúan como aislantes de la temperatura exterior.

En este tipo de preservación no se usa ninguna clase de insumo ni aditivo de conservación.

2. Pescado fresco refrigerado

Se da estrictamente en pesca de tipo comercial; se observó en los ríos U cayali, Marañón, Huallaga y Amazonas, en las embarcaciones denominadas "congeladoras", que tienen que desplazarse a distantes zonas de pesca.

Sus características principales son:

\section{- E species de mayor utilización}

Boquichico, palometa, sábalo, mojara, gamitana, lisa, yahuarachi, Ilambina, dorado, corvina y sardina.

\section{- Sistema de transporte}

En botes "congeladores".

\section{- L ugares de comercialización}

Y urimaguas, Pucallpa, N auta e Iquitos.

\section{- Tipo de refrigeración}

Refrigerado con hielo en trozos, que debe ser reemplazado en sus capas superficiales cada 2 días.

\section{- Forma de estibado}

En el cajón bodega" al granel con una base de hielo desmenuzado, de aproximadamente $15 \mathrm{~cm}$.; después de la última capa de hielo se cubre con plástico. Encima se le agrega una capa de cascarilla de arroz y finalmente se cierra herméticamente el cajón con una tapa de madera. 


\section{- Tiempo de conservación}

En el cajón (en las condiciones mencionadas) se va almacenando el pescado a medida que se captura, esto permite su conservación durante el tiempo que demora completar la capacidad de carga y el tiempo de transporte que, en totalçpuede ser de 10 a 1.5 días.

- Tipo de preservación

Hielo desmenuzado y complementado con un valioso auxiliar que es la cascarilla de arroz, que permite mantener la temperatura de trabajo y evitar la descongelación rápida del hielo.

- Cantidad de insumos utilizados

Hielo (45 panes/Tn. de pescado) y cascarilla de arroz (6 a 8 sacos).

- Medidas del cajón "bodega"

De 5 a $8 \mathrm{~m}$. de largo por 2 a 3 de ancho y 2 a $3 \mathrm{~m}$. de altura.

- Costo

Hielo a 1/. 15/pan.; cascarilla de arroz a 1/. 10 saco.*

(*) Intis de 1986.

- Volumen de desembarco en la Amazonía

Según datos estadísticos del MIPE, actualmente se tiene un volumen de desembarco de pescado refrigerado de 3,476.65 T.M. (promedio anual 1981-1986). Se ha considerado los volúmenes de desembarco realizados en los puertos de Iquitos, Y urimaguas, Requena, Caballococha, el Estrecho y Contamana.

\section{B. Conservación del escado or la técnica de seco salado}

En toda la zona evaluada, el excedente del pescado capturado es eviscerado y sometido a conservación por adición de sal, lo que origina una penetración de $\mathrm{CIN}$ a en el músculo del pescado y una pérdida de agua mediante deshidratación; en este intercambio se basa al principio de conservación por este método. 
Sus características principales son:

- Tipo de corte

Es longitudinal, por el dorso (corte mariposa), desde la cabeza a la cola.

- Forma de eviscerado y lavado

Las vísceras son eliminadas manualmente y para ayudar a la penetración de la sal se hace con el cuchillo incisiones superficiales a lo largo de los músculos.

L a limpieza de los residuos de sangre y mucus se hace con la misma agua del río.

- Especies utilizadas

Boquichico, yahuarachi, lisa, sábalo y paiche.

- Insumos utilizados

Sal y hojas (bijao y pan de árbol).

- Forma de utilización de la sal

Existen variaciones de acuerdo al tiempo que se quiera preservar; así, trabajando con $100 \mathrm{~kg}$. de pescado, se utiliza $50 \mathrm{~kg}$. De sal para una duración de cuatro (4) meses; para tres (3) meses se usa $40 \mathrm{~kg}$. De sal y para dos (2) meses 0 menos 25 $\mathrm{kg}$.

- Forma de auilamiento

El pescado salado se apila en rumas y se cubre con hojas de bijao o pandisho.

\section{- Forma de secado}

Se hace por exposición al sol, en cordeles, plataformas de madera o troncos de árboles., En esta etapa, el pescado es guardado durante la noche para preservar 10 de la lluvia y/o evitar pérdidas.

- Tiempo de conservación en sal

Un día. 
- Tiempo de secado 2 - 3 días.

- Duración de la conservación 3 - 4 meses.

- Costo

El saco de sal oscila de 1/. 90 a 1/. 120 (mayo 1986).*

(*) Intis de 1986.

- Observaciones

En los ríos Huallaga y M arañón se observó en algunos casos el uso de sal de mina con buenos resultados, ésta abunda en la zona del alto Huallaga (Pilluana); su bajo precio es muy importante para este tipo de preservación.

- Volumen de pescado seco-salado desembarcado en la amazonía

Según datos estadísticos se ha tenido un promedio anual de 3,148.98 T.M. para los años 1980-86; se ha considerado los volúmenes de desembarco en los puertos de I quitos, Y urimaguas, R equena, Caballococha y Contamana.

\section{Conservación del pescado por la técnica del salpreso}

Tiene características de corte y eviscerado similar a los utilizados para secosalado. Sus principales características son:

\section{C antidad de sal utilizada}

15 - $20 \mathrm{~kg}$. de sal para $100 \mathrm{~kg}$. de pescado.

\section{- Forma de conservación}

Después de ser agregada la sal, el producto es colocado en una canasta fabricada de la corteza del aguaje, comunmente denominada paneros, que tiene una capacidad aproximada de 40 - $50 \mathrm{~kg}$.; éstas se cubren con hojas de bijao, lo que permite una buena preservación de la temperatura del medio ambiente.

\section{- Duración de la conservación}

20 - 30 días. 


\section{- Volumen de pescado salpreso desembarcado en la amazonía}

Los datos estadísticos indican que existe un promedio anual de 800.74 T.M. (1980-1986); se ha considerado los puertos de Iquitos, Requena, Y urimaguas, el Estrecho y Contamana.

\section{Conservación del pescado por la técnica del secado}

Para este tipo de conservación se utiliza preferentemente a los peces pequeños. El tipo de corte y eviscerado son similares a los empleados en el secosalado y salpreso; su característica principal es que no se utiliza básicamente la sal (puede ser superficialmente) como elemento de conservación, sino la radiación solar.

Lo mencionado permite su conservación para 3 a 4 días.

\section{E. Conservación de camarones}

Se observó este tipo de conservación en el río U cayali, en las zonas de Flor de Punga, Iberia, Tamanco, Perlita, San Roque, Requena y Jenaro Herrera.

Sus características principales son:

\section{- Especies}

- Macrobrachium amazonicum

- Insumos utilizados

Sal.

\section{- Forma de urocesamiento}

El camarón es seleccionado y lavado con agua de río. Se siguen los siguientes pasos:

Se precocina a temperatura de ebullición, durante 4 a 5 minutos, con agregado de un $2 \%$ de sal. 
Se deja en enfriamiento hasta el escurrido total (1/2 hora).

Se tiende en maderas o calaminas para' exposición directa al-sol durante dos días.

Se almacena en bolsas plásticas para su venta.

\section{- Precio de venta}

20 a 30 intis/kg. (en zona de captura - junio 1986).

Observación

Este camarón ( $M$ acrobrachium amazonicum) tiene su época de abundancia en los meses de junio, julio, agosto y setiembre.

\section{CONCLUSIONES Y RECOMENDACIONES}

Los artificios de preservación del pescado fresco utilizados durante el tiempo que dura la captura de peces (rapicheros, peces en cautiverio e introducidos en el agua), así como los medios de transporte (hojas de bijao, yarina, shapaja y plátano) son adecuados y eficientes; para peces menores y medianos se recomienda a nivel artesanal, en capturas menores de 1,000 kg.

- A nivel artesanal, con capturas mayores de 2 T.M. y menores ae 5 T.M., se recomienda (para preservación del pescado fresco), la utilización de hielo en trozos, complementado por las excel entes propiedades aislantes de la cascarilla de arroz.

- L Las técnicas de conservación del pescado fresco (refrigerado) utilizadas por las embarcaciones de pesca comercial "congeladoras" son deficientes; su almacenamiento es a granel, sin estibar, sin limpieza adecuada (no se elimina el mucus y otras materias orgánicas que se impregnan en las branquias) y existe maltrato por excesiva presión (peso de pescado y hielo). Se recomienda la aplicación de normas técnicas de manipulación y preservación de pescado a bordo por un técnico o especialista embarcado con poder de decisión.

- Otra limitación que tiene el empleo de la técnica de refrigeración, utilizadas por las embarcaciones de pesca comercial "congeladoras" es que solamente es aplicable para un tiempo máximo de 15 -20 días, considerando que es política del sector pesquero ampliar las zonas de pesca hacia áreas productivas que permiten la captura durante todo el año. Para desplazamientos más distantes, se 
recomienda iniciar estudios tendientes a dotar a las embarcaciones de pesca comercial, con bodegas equipadas con sistemas de çongelación para conservación del pescado por períodos de hasta 2 meses.

Las técnicas artesanales de conservación de pescado (sal preso y seco- salado) son realizadas en general sin parámetros técnicos establecidos: asimismo, los porcentajes de sal utilizados son bastante variables. Se recomienda realizar ensayos para mejorar y uniformizar las fases del proceso en cada caso. (Por especie y por tamaño), así como analizar bromatológicamente y microbiológicamente los productos en conservas que se venden en los mercados de Iquitos y Pucallpa.

U no de los grandes problemas para la conservación del pescado por la técnica del salpreso y seco-salado es la disponibilidad de sal, que alcanza al tos precios en los ríos; la utilización en forma industrial de los depósitos de sal que se encuentran en el alto Huallaga sería la solución, ya que este tipo de sal posee excelentes propiedades para el tratamiento de productos pesqueros.

El camarón amazónico (M acrobrachium amazonicum) una especie que abunda en grandes cantidades en el río U cayali, se recomienda su utilización en conservas. La técnica es bastante simple y el producto final de alto costo.

Según datos estadísticos de la dirección de extracción en salpreso y secosalado, las especies de mayor utilización son: el boquichico, el yahuarachi, el paiche, la gamitana, la doncella, la arahuana, la lisa, la palometa, el sábalo, la ractacara y el maparate.

El pescado utilizado para el consumo humano al estado fresco (refrigerado) se ha incrementado entre los años de 1981-1986 en un 14\%, siendo los volúmenes de desembarque anuales los siguientes: 1,739.6 T. M ., 2,601.13 T.M., 3,578.19 T.M., 3,709.46 T.M. y 3,906.58 T.M.

El pescado al estado seco-salado se ha incrementado en 5 años (1980-1985) en un $21.33 \%$, siendo los volúmenes de desembarque los siguientes: 389. 9 T.M., 1,313.85 T.M ., 2,106.50 T.M., 1,971.99 T.M. y 7,846.62 T.M .

El pescado desembarcado al estado de salpreso se ha incrementado en 5 años (1981-1985) en un 19.70\%, siendo los volúmenes de desembarque los siguientes: 90.00 T.M., 401.22 T.M., 555.51 T.M., 517.47 T.M. y 548.57 T.M.

Los resultados permiten asegurar que existe un significativo excedcnte de pescado que es conservado a nivel artesanal. Finalmente se recomienda realizar 
estudios tendientes a obtener los factores de conversión por especie de salpreso y seco-salado a valores reales de materia prima.

\section{BIBLIOGRAFIÁ}

1. Instituto de Investigaciones de la A mazonía Peruana-IIA P (1987). Documento de trabajo No 026-87-DIT. Estudio sobre extracción y conservación de recursos pesqueros de la amazonía. Iquitos, Perú. 84 pág.

2. Instituto de Investigaciones de la Amazonía Peruana -IIAP (1984). Diagnóstico de la pesquería en la región amazónica. Loreto-U cayali. IquitosPerú. 128 pág.

3. M inisterio de Pesquería (1987). Datos estadísticos de volumen de desembarco de pescado en Loreto. 1981-1986. Iquitos-Perú. "s.p.'

4. M inisterio de Pesquería (1982). M anipulación y preservación de pescado. 1ra. Ed. Lima. 97 pág. 